\title{
Signal-Amplified Colorimetric In Situ Hybridization for Assessment of Human Papillomavirus Infection in Cervical Lesions
}

Peter Birner, M.D., Barbara Bachtiary, M.D., Bettina Dreier, Ph.D., Monika Schindl, M.D., Elmar A. Joura, M.D., Gerhard Breitenecker, M.D., Georg Oberhuber, M.D.

Institute of Clinical Pathology, Department of Gynecopathology and Cytology (PB, MS, GB, GO); Department of Radiotherapy and Radiobiology (BB, BD); and Department of Gynecology and Obstetrics (EAJ), University of Vienna, Austria

Detection and typing of human papillomavirus (HPV) infection may have a major impact in cervical-screening and follow-up. In this study various commercially available techniques for the detection of HPV were evaluated. HPV-status was determined in 86 samples of cervical cancer by PCR and direct sequencing, catalyzed signal amplified colorimetric DNA in situ hybridization (CSAC- ISH) (GenPoint system, DAKO), immunohistochemistry (IHC) and in 12 selected cases also by conventional, non-amplified ISH. Twenty-one samples of cervical intraepithelial neoplasias grade III (CIN III) were investigated by CSAC-ISH, conventional ISH and by IHC, in corresponding PAP smears HPV-detection and typing was performed by CSAC-ISH and Hybrid Capture test II (HC). In additional 20 PAP smears HPV typing was performed using $\mathrm{HC}$ and a novel immunocytochemical system for HPV detection and-typing. CSAC-ISH showed good correlation with PCR analysis in cervical cancers: In $87 \%$ of PCR positive cases, HPV infection was also detected by CSAC- ISH (66/76). HPV 16 was detected in $75 \%$ of PCR-positive cases (44/59), HPV 18 in $71 \%$ of PCR positive cases (5/7). CSAC-ISH detected HPV 31 in only $29 \%$ of PCR positive cases (2/7), and HPV 33 in $64 \%$ of PCR-positive cases (23/36). Nevertheless, CSAC-ISH- false negative cases for HPV 31 or 33 were nearly always combined infections with other HPV types, which were detectable by CSAC-ISH in most cases. CSAC-ISH revealed HPV infection in 20 of 21 HC-positive cervical smears, while in corre-

Copyright () 2001 by The United States and Canadian Academy of Pathology, Inc.

VOL. 14, NO. 7, P. 702, 2001 Printed in the U.S.A.

Date of acceptance: February 27, 2001.

DAKO reagents were provided by DAKO Austria; KREATCH reagents by Zamponi Diagnostics, Austria; Viroactiv system by Virofem Diagnostica, Germany.

Dr. Peter Birner, University of Vienna, Institute of Clinical Pathology, Währinger Gürtel 18-20, A-1090 Vienna, Austria; e-mail: peter.birner@ akh-wien.ac.at; fax: 43-1-405-3402. sponding biopsies (CIN III) CSAC-ISH detected $100 \%$ of HPV infections. Conventional, nonamplified ISH showed significantly lower sensitivity compared with CSAC-ISH, and immunocyto- and -histochemistry were of very low sensitivity for detection of HPV. CSAC-ISH is an easy-to-handle method for detection and typing of cervical HPV infection, and shows sufficient sensitivity for clinical practice.

KEY WORDS: Human papillomavirus, cervical cancer, In situ hybridization, polymerase chain reaction, signal amplification, hybrid capture test.

Mod Pathol 2001;14(7):702-709

Infection of the cervical epithelium by human papillomavirus (HPV) is considered to support the oncogenic process leading to the development of cervical cancer (1), one of the most common cancers in women worldwide (2). The risk of developing cancer is dependent on the HPV type present within the epithelial cells. According to their oncogenic potential HPV may be classified in a low oncogenic risk group (i.e., HPV 6, 11, 42, 43, and 44) and in an intermediate or high oncogenic risk group (i.e., HPV 16, 18, 31, 33, 35, 39, 45, 51, 52, 56, $58,65,66)(1)$.

Although the majority of patients with dysplastic cervical epithelium shows infection with HPV of any type, complementation of conventional morphologic analysis with identification of the virus by sensitive techniques may support further clinical decisions (3). Also, it was proposed that HPVdetection and typing should support cytological screening $(4,5)$. These facts explain, why a great interest in HPV- detection and typing does exist (6). Several methods are available for this task: At the moment, the hybrid capture II test system (HC) (Digene, Silver Spring, Md) is considered the gold standard for HPV testing in clinical practice (7). 
Although this system is reliable, sensitive and easy to handle $(1,2)$, it has several disadvantages: It can only be performed in cervical smears, but not in paraffin- embedded, archived material. In addition, no exact typing is performed, HPV infection is attributed to a "low-risk" or "high-risk" group. Another major disadvantage are the high costs of this system (3).

Polymerase chain reaction (PCR) has an extremely high sensitivity and might be performed on smears as well as on paraffin- embedded material (1). The major disadvantage of this system is that it is always confronted with the problem of contamination. Also, a high laboratory infrastructure is required (8).

Immunocyto- and -histochemistry are cheap, easy-to handle methods for HPV-detection. However, the sensitivity is low, and typing was considered problematic up to now. Whether this opinion will change after the introduction of a new immunocytochemical system for HPV detection and typing (Viroactiv; Virofem Diagnostica GmbH, Mainz, Germany) is unclear as only few data on the performance of this system have been published so far (9).

In situ hybridization (ISH) is an easy to handle, reliable method for HPV detection and typing, working on PAP-smears and paraffin-embedded sections $(10,11)$. Its main problem was the low sensitivity. The detection limit of both radioisotopic and nonisotopic labels in genomic probes is 10 to 50 viral copies per cell in formalin-fixed samples $(12,13)$. Unfortunately, nearly $50 \%$ of cervical cancers with HPV 16 infection have 50 or less viral copies per infected cells, with a peak between 1 and 10 copies (14). Therefore, several attempts have been undertaken to enhance sensitivity of ISH (12).

A nonisotopic, colorimetric signal amplification system for ISH is now commercially available (GenPoint system, DAKO, Glostrup, Denmark) (15). This system is based on the catalyzed amplification of positive hybridization signals using biotin-tyramide complexes (16) and does not require PCR amplification or the use of radioactive labeled probes. Recently, similar, not commercially available signal amplified ISH systems have been used to identify HPV 16 specific sequences in infected cell lines and in cervical neoplastic lesions with high sensitivity, even capable of detecting single viral copies in routinely fixed material $(12,17)$.

The aim of our study was to compare the sensitivity and specificity of PCR, HC assay and a catalyzed signal amplified colorimetric (CSAC) ISHsystem in PAP smears and in paraffin-embedded sections of cervical cancers and severe cervical dysplasias. In addition, the possibility of HPV detection and typing using immunocyto- and -histochemistry was investigated.

\section{MATERIALS AND METHODS}

\section{Cervical Cancer Group}

In this group HPV-detection and/or typing was performed using PCR, CSAC-ISH and immunohistochemistry (IHC). 86 consecutive biopsies with invasive cervical cancers FIGO stage IB to IIIB (8 cases IB, 6 IIA, 35 IIB, 11 IIIA, 26 IIIB) were retrieved from our files. For inclusion into this study sufficient material had to be available on the tissue blocks.

\section{Cervical Dysplasia Group}

In addition, 21 PAP smears (diagnosed as highgrade squamous intraepithelial lesions) of patients with known HPV high-risk infection, assessed by HC (see below), were included in our study. All patients had also undergone cervical biopsy, in which severe cervical dysplasia/carcinoma in situ (CIN III) was evident. HPV-detection in cervical smears was performed by CSAC-ISH, in corresponding biopsy specimens by CSAC-ISH, conventional ISH and IHC.

In addition, we investigated cervical smears of 20 patients out of a high-risk collective with the Viroactiv system (Virofem Diagnostica GmbH, Mainz, Germany), an immunocytochemcial system consisting of screening antibody and an antibody considered specific for high- risk types only. For comparison, patients were also investigated with HC.

\section{Human Papillomavirus Analysis}

PCR

Ten-micrometer-thick paraffin-embedded tissue sections were treated with xylene to remove the paraffin and subsequently washed with ethanol. Sections were digested with proteinase K $(100 \mu \mathrm{g} /$ $\mathrm{mL}$ ) for 4 hours at $56^{\circ} \mathrm{C}$, followed by inactivation of the enzyme by heating the tubes in a thermocycler for $8 \mathrm{~min}$ at $94^{\circ} \mathrm{C}$. Extracted DNA was processed immediately or frozen at $-20^{\circ} \mathrm{C}$ until further investigation. After every tenth specimen negative controls consisting of liver, lung, heart, and brain were included and subjected to subsequent HPV PCR analysis.

PCR was performed in $50 \mu$ l-reaction tubes containing $50 \mathrm{~mm}$ KCL, $10 \mathrm{~mm}$ TRIS ( $\mathrm{pH}$ 9.0), $0.1 \%$ Triton X-100, $100 \mu \mathrm{M}$ dNTP's, $1.5 \mathrm{~mm} \mathrm{MgCl} 2,1.5 \mathrm{U}$ of Taq-DNA polymerase and 50 pmol of each primer. During each amplification a positive control (extracted DNA from HeLa cells) and a negative control (water) were carried out. All samples were tested for $\beta$-globin (PC03/Pc04: 110bp); 18), which served as internal control for integrity of the extracted template, followed by consensus HPV-PCR $(\mathrm{GP} 5+/ \mathrm{GP} 6+)$; 19) from the L1 region. Typing of HPV DNA was performed by type-specific PCR: 
HPV16 (E7/E1: 698-917), HPV18 (E6/E7: 533-705), HPV31 (E5: 3835-3989), HPV33 (E6: 265-396), HPV35 (E7: 610-840) and HPV45 (E6/E7: 548-694; 20, 21).

Cycling parameters for all samples were: $50 \mathrm{sec}$ denaturation at $94^{\circ} \mathrm{C}, 50 \mathrm{sec}$ annealing at $60^{\circ} \mathrm{C}$ (except for HPV consensus and HPV 33 annealing at $\left.50^{\circ} \mathrm{C}\right), 75 \mathrm{sec}$ extension at $72^{\circ} \mathrm{C}(40$ cycles $)$ and 5 min at $72^{\circ} \mathrm{C}$ for final extension.

After PCR amplification, the PCR-products were separated on a $2 \%$ TAE agarose gel followed by visualization of the DNA fragments under UV light.

To detect infection with other HPV-types, consensus PCR-products were cut out and purified using the QIAquick Gel Extraction kit (Qiagen, Hilden, Germany) according to the manufacturer's protocol. The purified consensus HPV DNA was cloned into pTargeTTM Mammalian Expression Vector System (Promega, Madison, USA), followed by thermo-cycle sequencing (Amersham Life Science, Ohio, USA) according to the manufacturer's instructions.

\section{ISH}

ISH was performed in paraffin-embedded specimens fixed in $4 \%$ buffered formalin and in archived PAP smears (up to 2 years of age). Histological slides, $4 \mu \mathrm{m}$ in thickness, were deparaffinized in xylene. CSAC- ISH was performed as follows: Target retrieval of histological sections was performed with Target Retrieval Solution (\#S1700, DAKO, Glostrup, Denmark) in a waterbath at $95^{\circ} \mathrm{C}$ for 40 minutes, followed by digestion with $0.01 \%$ pepsin in $0.2 \mathrm{M} \mathrm{HCl}$ for 13 minutes. Coverslips of PAP smear slides were removed in xylene, and destaining with $2 \mathrm{~m} \mathrm{HCl}$ for 5-15 minutes under microscopic control was performed. Background quenching in all specimens was performed by $0.3 \%$ $\mathrm{H}_{2} \mathrm{O}_{2}$ for 20 minutes. $15 \mu \mathrm{L}$ of the biotinylated probes for general screening (HPV 6, 11, 16, 18, 30, 31, 33, 45, 51, 52) (\#Y 1404, DAKO) and type specific probes against HPV 16, 18, 31 and 33 (\#Y 1407, Y 1408, Y 1409 and Y1410, all DAKO) were applied on separate sections of each paraffin- embedded specimen, and were covered with coverslips. ISH on PAP smears was performed with the screening probe only. Target and probe DNA was denaturated by heating the slides for $90^{\circ} \mathrm{C}$ for 5 minutes in an oven. Hybridization was performed in a humid chamber at $37^{\circ} \mathrm{C}$ overnight. After washing, the GenPoint Kit (\#K 0620; DAKO) was used according to the manufacturer's instruction: First, primary Streptavidin- HRP in a dilution of 1:200 was incubated for 15 minutes. After washing, biotintyramide solution was incubated for $15 \mathrm{~min}$, followed by the secondary streptavidin-HRP for 15 min.
To compare sensitivity of CSAC- ISH with conventional, non-amplified ISH, HPV detection also was performed using the Rembrandt ISH Kit (KREATECH Diagnostics, Amsterdam, The Netherlands) according to the manufacturer's instructions in all tissue samples of CIN III and in 12 samples of invasive cancer with low number of (integrated) viral copies ( 1 to 2 signals per nucleus) at CSACISH using the screening probe.

HPV typing in samples of CIN III was performed with 2 biotinylated probes, capable of detecting HPV 16/18 DNA (\#PLB008; KREATECH; region: 7.9 Kb HPV 16 and $7.9 \mathrm{~Kb}$ HPV 18 total genomes) and HPV 31/33 DNA (\#PLB004; KREATECH; region: 7.9 Kb HPV 31 and 7.9 Kb HPV 33 total genomes). HPV detection in cervical cancer specimens was performed using a biotinylated panHPV probe (\#PLB000; KREATECH; region: mix of total genomes $(7 \mathrm{~K}-8 \mathrm{~Kb})$ and DNA containing the conserved HPV region (200 bp)). Hybridization was performed in a humid chamber at $37^{\circ} \mathrm{C}$ overnight. With both systems, visualization of positive hybridization signals was performed by incubation with diaminobenzidine for 8 minutes. Conterstaining was performed with hematoxylin.

For positive and negative control, control slides with HPV-infected cervical cancer cell lines provided by the manufacturers in the various kits were used.

As additional negative control of CSAC- ISH on PAP smears, smears from the oral cavity of 2 healthy persons were treated in the same way as PAP smears (including removal of coverslips in xylene and destaining).

\section{Hybrid Capture Test}

The HPV status in cervical smears was determined with the commercially available hybrid capture test II (HC) (Digene, Silver Spring, Md) (3). The assay kit contains two specific RNA-probes, one for low-risk HPV-types $(6,11,42,43,44)$ and one for high-risk types $(16,18,31,33,35,39,45,51,52$, 56, $58,59,68$ ), which hybridize with appropriate DNA, gained from a sample collected with a brush from the patient's cervix. The hybrid is bound by polyclonal antibodies which are fixed in the testing tube. After applying a secondary monoclonal antibody marked by a chemoluminescent substance, hybrids are detected luminometrically (2).

\section{Immunohisto- and -Cytochemistry}

Immunohistochemically HPV-detection in $4 \mu \mathrm{m}$ section of paraffin embedded cervical biopsies was performed using the monoclonal antibody K1H8 (DAKO, Glostrup, Denmark) (22), capable of detecting a main capsid-epitope of HPV $6,11,16,18,31$, 33, 42, 51, 52, 56, and 58. Histological slides were deparaffinized in xylene. Target retrieval was per- 
formed with Target Retrieval Solution (\#S1700, DAKO, Glostrup, Denmark) in a microwave oven for 30 minutes. Background quenching was performed by $0.3 \% \mathrm{H}_{2} \mathrm{O} 2$ for 20 minutes. The primary antibody was incubated for 1 hour in a dilution of 1:50 at room temperature, detection was performed using the ChemMate kit (DAKO, Glostrup, Denmark) and diaminobenzidine.

As positive control a specimen of CIN III with HPV 16 infection determined by ISH and known positive immunoreactivity was used.

For immunocytochemical HPV- detection and -typing in cervical smears, the Viroactiv system (Virofem Diagnostica GmbH, Mainz, Germany) was used according to the manufacturer's instructions. This system consists of two monoclonal anti-HPV antibodies: The screening antibody S33 detects a highly conserved epitope on the L1 protein, while the screening antibody T16 detects a N'terminal epitope of L1 and is considered to detect HPV 16, 18,33 and 39. Cervical scrapes were collected using a brush after patients had given an informed consent, and transferred into a fixation solution. This solution was transferred to special slides and fixed with methanol.

Antigen retrieval was performed in citrate buffer in a microwave oven. Specimens were incubated overnight at $+4^{\circ} \mathrm{C}$ with the primary antibodies. Detection was performed with an APAAP system provided in the kit.

\section{RESULTS}

\section{Cervical Cancer Group}

PCR analysis revealed HPV-infection in 76 samples of cervical cancer (88.4\%) (Fig. 1). In 37 cases (43\%) infection with only one HPV-type was found, but in 39 cases $(45.3 \%)$ two or more HPV types were detected. In 59 cases (68.6\%) HPV 16 was observed, in 7 patients (8.1\%) HPV 18 or HPV 31, respectively,

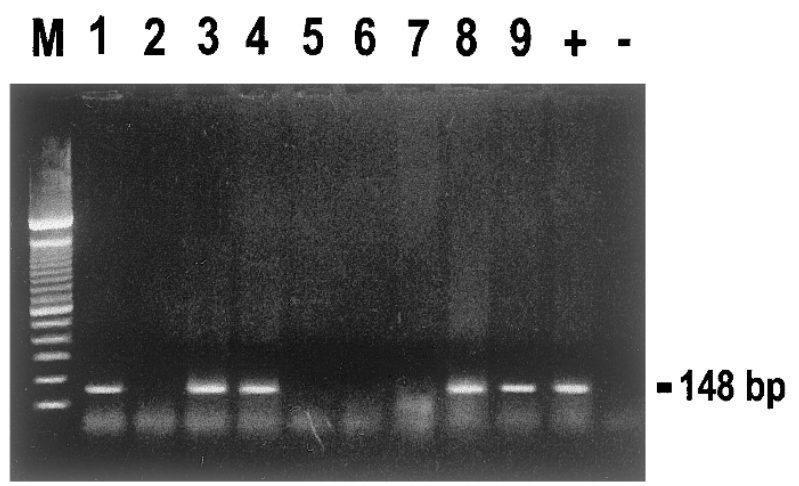

FIGURE 1. HPV consensus primer PCR for the L1 region (148bp) in samples of cervical cancer. $\mathrm{M}=\mathrm{DNA}$ molecular weight marker. Positive PCR- reactions in specimens $1,3,4,8,9 .+=$ positive control, - = negative control. in 36 subjects (41.9\%) HPV 33, in 6 patients (7\%) HPV 45 and, finally, HPV 35, 69 or 73 were detected in 1 one case (1.2\%) each, and HPV 58 in 2 patients (2.3\%).

The negative controls were free from artifacts when CSAC- ISH was performed. Two different patterns of positive signals were observed in positive samples: a patchy signal unevenly distributed over tumor cell nuclei (Fig. 2A), representing episomal HPV-DNA (23), and a dot- like signal in tumor cells (Fig. 2B), representing integrated HPV-DNA (24).

CSAC- ISH showed very good correlation with PCR for general detection of HPV and for detection of HPV 16 and 18 (results complied in Table 1). Interestingly, one case which was HPV-negative at PCR, showed positive CSAC- ISH with the screening probe. This case showed no positive signal at subsequent CSAC- ISH with probes against HPV 16, 18, 31, and 33. Unfortunately, there was not enough material left for further investigation.

Sensitivity for detection of HPV 31 was low, but all 5 false negative cases showed infection with other HPV-types, and CSAC-ISH with the screening probe was positive in all cases. Positive CSAC- ISH for another HPV type was obtained in 2 of the 4 cases, where at least one probe against the additional HPV types was available.

CSAC- ISH detected HPV 33 in $64 \%$ of PCR positive cases. The results were similar to those obtained with HPV 31 probes: All 13 false negative cases in CSAC- ISH showed combined infection with other HPV-types at PCR. CSAC- ISH using the screening probe was positive in 11 of these 13 cases. In 7 of these 11 cases, positive CSAC- ISH was obtained with the probe against HPV 16.

\section{Cervical Dysplasia Group}

In cervical smears, in 20 of 21 patients (95.2\%) showing HPV infection in HC, positive results of CSAC- ISH using the screening probe were obtained. The staining pattern was slightly different compared with CSAC- ISH on paraffin embedded specimens, since positive signals were also obtained in the cytoplasm of cells (Fig. 2C). Assessment of CSAC- ISH in PAP smears was more difficult than in paraffin embedded specimens, due to overlaying of cells. In the corresponding biopsy specimens, a positive reaction with the CSAC- ISH screening probe was observed in all 21 cases. Sensitivity of CSAC-ISH in our collective of 21 PAP smears was $95.2 \%$ for general detection of HPVinfection. Type specific CSAC- ISH revealed infection with HPV 16 in 14 cases (66.7\%), HPV 18 in 1 case $(4.8 \%)$, HPV 31 in 4 cases $(19 \%)$, and HPV 33 in 9 (42.9\%). In only one case, no infection with a certain HPV type was found with the available ISH-probes, suggesting an infection with a HPV- 
high-risk type that is detected by $\mathrm{HC}$ and the ISH-screening probe, but for which no specific ISHprobe was available.

\section{Comparison of Conventional ISH and CSAC-ISH}

Signal intensity was generally weaker in positive cases at conventional ISH compared with CSACISH (Fig. 2E\& 2F). Positive cells were found mainly at the surface of the dysplastic epithelium. Nonamplified, conventional ISH was of significantly lower sensitivity compared CSAC-ISH: At HPVtyping, the HPV 16/18 probe detected 7 of the 14 cases $(50 \%)$ with positive signal at CSAC- ISH for HPV 16, the case positive for HPV 18 at CSAC- ISH was not detected. Of the 9 cases positive for HPV 33 at CSAC- ISH, conventional ISH detected only one
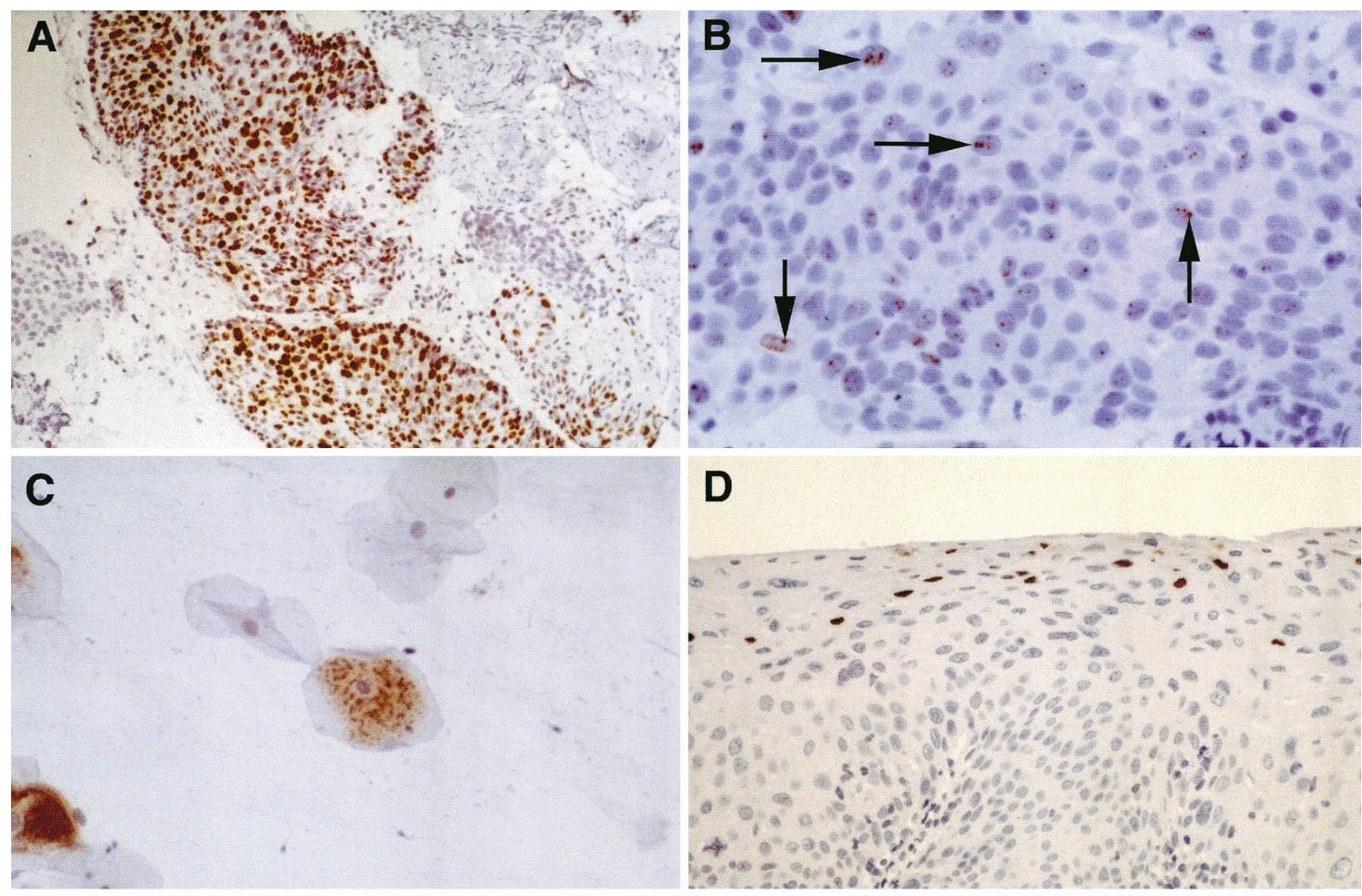

D
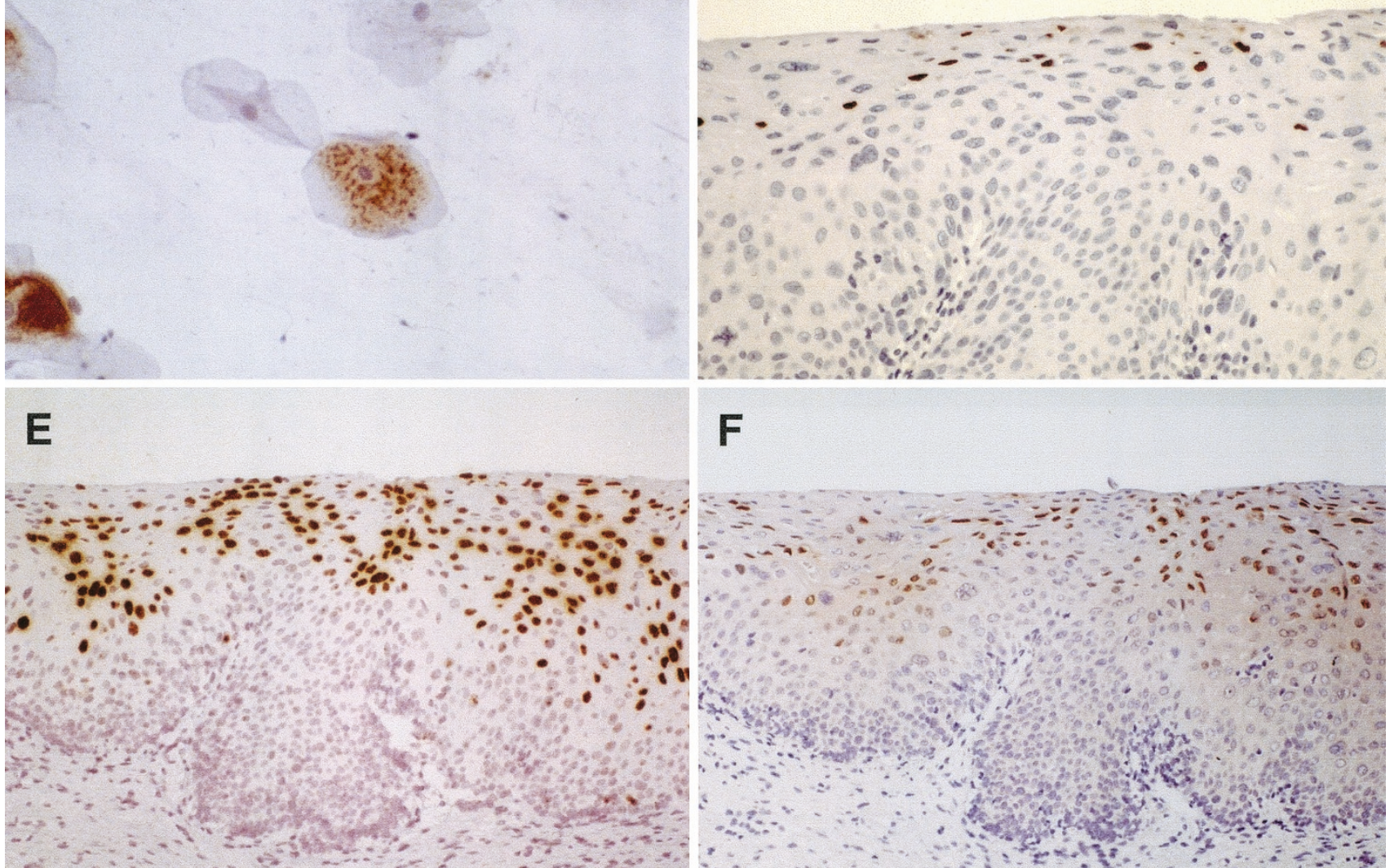

\section{$\mathbf{F}$}

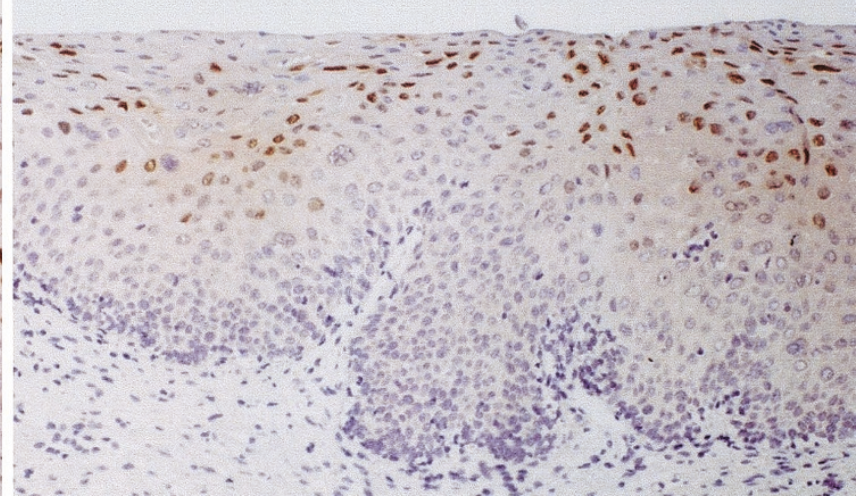

FIGURE 2. A) Sample of cervical cancer with high (episomal) viral load, detected by CSAC- ISH and HPV-screening probe. Original magnification, $40 \times$. B) Sample of cervical cancer with low (integrated) viral load detected by CSAC- ISH and HPV-screening probe. Arrows indicate samples of typical dot-like positive signals in nuclei. Original magnification, 300×. C) Positive signal obtained in a cervical smear detected by CSAC- ISH and HPV- screening probe. Note the strong presence of cytoplasmic HPV-copies. Original magnification, 400×. B) Immunohistochemical detection of HPV capsid protein in a sample of CIN III (same as in Fig. 2E and 2F). Immunoperoxidase, original magnification x200. E) Sample of CIN III (same as in Fig. 2D and 2F), HPV infection detected by CSAC-ISH using a probe vs. HPV 16. Original magnification x200. F) Sample of CIN III (same as in Fig. 2D and 2E), HPV infection detected by conventional, non-amplified ISH using a probe vs. HPV 16/18. Note the smaller number of nuclei decorated and the weaker signal intensity compared with CSAC-ISH in Fig. 2E. Original magnification x200. 
TABLE 1. Correlation of PCR and CSAC-ISH for Detection and Typing of HPV Infection in Samples of Cervical Cancer $(n=86)$

\begin{tabular}{|c|c|c|}
\hline & \multicolumn{2}{|c|}{$\begin{array}{l}\text { PCR-Consensus Primer for } \\
\text { High-Risk Types }\end{array}$} \\
\hline & Positive & Negative \\
\hline \multicolumn{3}{|c|}{ ISH-screening probe } \\
\hline Positive & $66(87)$ & $1(10)$ \\
\hline \multirow[t]{2}{*}{ Negative } & $10(13)$ & $9(90)$ \\
\hline & \multicolumn{2}{|c|}{ PCR-HPV-16 } \\
\hline \multicolumn{3}{|c|}{ ISH-probe HPV 16} \\
\hline Positive & $44(75)$ & - \\
\hline \multirow[t]{2}{*}{ Negative } & $15(25)$ & $27(100)$ \\
\hline & \multicolumn{2}{|c|}{ PCR-HPV 18} \\
\hline \multicolumn{3}{|c|}{ ISH-probe HPV 18} \\
\hline Positive & $5(71)$ & - \\
\hline \multirow[t]{2}{*}{ Negative } & $2(29)$ & $79(100)$ \\
\hline & \multicolumn{2}{|c|}{ PCR-HPV 31} \\
\hline \multicolumn{3}{|c|}{ ISH-probe HPV 31} \\
\hline Positive & $2(29)$ & - \\
\hline \multirow[t]{2}{*}{ Negative } & $5(71)$ & $79(100)$ \\
\hline & \multicolumn{2}{|c|}{ PCR-HPV 33} \\
\hline \multicolumn{3}{|c|}{ ISH-probe HPV 33} \\
\hline Positive & $23(64)$ & - \\
\hline Negative & $13(36)$ & $50(100)$ \\
\hline
\end{tabular}

PCR, polymerase chain reaction; CSAC-ISH, catalyzed signal amplified colorimetric DNA in situ hybridization; HPV, human papillomavirus.

case $(11 \%)$. In HPV-infections in samples of CIN III detected by conventional ISH CSAC- ISH revealed a high (mainly episomal) viral load. All cases of CIN III with immunohistochemical detectable HPVinfection showed also detectable HPV-infection in conventional ISH.

In none of the 12 cervical cancer samples with low number of viral copies at CSAC- ISH a positive signal was obtained by means of conventional, non-amplified ISH.

\section{Immunohisto- and -Cytochemistry}

In none of the 86 samples of cervical cancer HPV capsid protein was detected by means of IHC (DAKO antibody). In the 21 samples of cervical dysplasias, positive immunostaining was obtained in only 3 cases (14.3\%) (Fig. 2D).

With the Virofem-System, no positive reaction was obtained in any patient, although the provided positive control always demonstrated distinct nuclear staining. 15 of the 20 patients showed HPVhigh- risk infection in the HC-assay. Since even with the help of the manufacturer no positive staining was obtained in any of these patients at repeated trials, this system was excluded from any further consideration.

\section{DISCUSSION}

In this study we have evaluated the presently commercially available methods for the detection of HPV infection in cervical smears and formalin-fixed, paraffin- embedded samples of cervical lesions. It is well known, that owing to their expertise in morphology pathologists prefer methods that allow visualization of a reaction product in combination with histomorphology. Our main goal was, therefore, to analyze the performance of a commercially available ISH -system using a novel signal amplification method (CSAC). Results were compared with those of PCR and HC. The specimens analyzed included also specimens with advanced cervical cancer. This was done owing to the fact that the number of HPV-copies may be very low in such a collective, in particular in advanced cases (14), thus challenging the sensitivity of the methods used.

Up to now, conventional ISH for detection of HPV infection was handicapped by its low sensitivity, as was shown by several earlier studies (25) and also in this study. One of the major argument in favor of ISH is that it is the only method, except in situ PCR, by which the viral genome can be identified in topographical relation to the pathological lesion (26). Although in situ PCR is considered highly sensitive (27), it is still a controversial method due to poor reproducibility and production of artifacts (28). In our personal experience it is extremely difficult to handle, and definitely not suitable for HPV-detection and -typing in a routine pathological laboratory.

In contrast to conventional ISH, CSAC- ISH showed a very good correlation with PCR as well in the detection of HPV genome with a general screening probe as well as with HPV type specific probes. The only exception was a low sensitivity of CSACISH to detect the genome of HPV 31 and to a minor extent also of HPV 33. One explanation might be that most patients which were falsely HPV 31 or HPV 33 negative, suffered from a combined infection with other HPV-types, possibly keeping the copy number of HPV 31 and 33 very low. Nevertheless further studies are required, since to our knowledge, no data exist on this subject. Interestingly enough, the other co-infecting types where detectable by ISH in most cases. Therefore, overall the performance of CSAC- ISH was satisfactory in particular when keeping in mind that the diagnosis of an infection with HPV-high-risk types is of central importance, and only to a lesser degree which HPVhigh-risk types are involved.

Certainly, in the future, even more sensitive amplification methods will be developed. E.g., sensitivity of signal amplification using biotinylated tyramide might be even increased by repeating the amplification step several times, but this procedure 
must be carefully established due to the possibility of artifacts.

Sensitivity of the CSAC- ISH using the screening probe in cervical smears was similar to HC. Nevertheless, ISH is handicapped by the fact, that on a traditional PAP smear only one ISH can be performed, i.e., screening for HPV in our case. Probably, using a cocktail of ISH- probes recognizing either high-risk or low-risk HPV types may render HPV typing on traditional PAP-smears suitable. Another disadvantage of ISH on PAP-smears is the fact that interpretation can be difficult due to overlaying of cells. This could be avoided by using liquid based cytology- specimens for ISH. With this method also several specimens from one smear might be produced, thus enabling the pathologist to apply various type specific probes. The major disadvantage of liquid based cytology is the increase in costs.

Our finding of a low sensitivity of IHC for detection of HPV-infection in high grade cervical lesions is in good correlation to other authors (11). To our best knowledge, we are the first to show the low sensitivity of IHC in detection of HPV in cervical cancer. One major disadvantage of IHC is the fact that the antibodies are directed against capsid proteins, which are only found in productive, but not in latent infection (11), thus explaining in part the low sensitivity of this method.

In conclusion, CSAC- ISH is a sensitive, easy to handle method for HPV-detection and -typing in cervical squamous intraepithelial lesions and cancers, highly suitable for routine pathology. It is also highly sensitive for detection of HPV-infection in traditional PAP smears, although interpretation is sometimes difficult and typing is problematic due to the lack of material. CSAC-ISH is now used in our laboratory in addition to HC for HPV-detection and -typing in routine specimens, especially in paraffinembedded cervical and also vulvar biopsies, and has proved as simple and reliable method.

In contrast to CSAC- ISH, our study confirms that immunohisto- and -cytochemistry are not suitable for detection and typing of HPV-infection in cervical smears and in paraffin-embedded sections of cervical dysplasias and cancers.

Acknowledgments: The authors thank Ms. Beatrix Reiter and Ms. Ankica Krmpotic-Salama for excellent technical assistance and Dr. Thomas Ströbel for critical discussion of the manuscript. The help of Ms. Andrea Güthlin-Dörfler and Ms. Josefine Stani was highly appreciated.

\section{REFERENCES}

1. Nindl I, Greinke C, Zahm D, Stockfleth E, Hoyer H, Schneider A. Human papillomavirus distribution in cervical tissues of different morphology as determined by hybrid capture assay and PCR. Int J Gynecol Pathol 1997;16:197-204.

2. Recio FO, Sahai-Srivastava BI, Wong C, Hempling RE, Eltabbakh GH, Piver MS. The clinical value of digene hybrid capture HPV DNA testing in a referral-based population with abnormal pap smears. Eur J Gynaecol Oncol 1998;19:203-8.

3. Birner P, Schindl M, Stani J, Oberhuber G, Czerwenka K, Vutuc C, et al. Hybrid capture based human papillomavirus typing in cervical screening compared to cytology and histology. Wien Klin Wochenschr 2000;112:761-6.

4. Schneider A, Zahm DM, Kirchmayr R, Schneider VL. Screening for cervical intraepithelial neoplasia grade 2/3: validity of cytologic study, cervicography, and human papillomavirus detection. Am J Obstet Gynecol 1996;174:1534-41.

5. Meijer CJ, Helmerhorst TJ, Rozendaal L, van der Linden JC, Voorhorst FJ, Walboomers JM. HPV typing and testing in gynaecological pathology: has the time come? Histopathology 1998;33:83-6.

6. Breitenecker G, Birner P. Zervixzytologie-quo vadis? Geburtshilfe Frauenheilkd (in press).

7. Clavel C, Masure M, Bory J, Putaud I, Mangeonjean C, Lorenzato M, et al. Hybrid Capture II-based human papillomavirus detection, a sensitive test to detect in routine high-grade cervical lesions: a preliminary study on 1518 women. Br J Cancer 1999;80:1306-11.

8. Victor T, Jordaan A, du Toit R, Van Helden PD. Laboratory experience and guidelines for avoiding false positive polymerase chain reactions. Eur J Clin Chem Clin Biochem 1993; 31:531-5.

9. Griesser H, Zeck S, Rausch-Damovsky M. Immunochemical detection of high-risk mucosal human papillomavirus infection in routine cervical smears [abstract]. Pathol Res Pract 2000;196:443.

10. Nuovo GJ. Detection of human papillomavirus in Papanicolaou smears: correlation with pathologic findings and clinical outcome. Diagn Mol Pathol 1998;7:158-63.

11. Delvenne P, Fontaine M, Delvenne C, Nikkels A, Bonvier J. Detection of human papillomaviruses in paraffin-embedded biopsies of cervical intraepithelial lesions: analysis by immunohistochemistry, in situ hybridization, and the polymerase chain reaction. Mod Pathol 1994;7:113-9.

12. Zehbe I, Hacker GW, Su H, Hauser-Kronberger C, Hainfeld JF, Tubbs R. Sensitive in situ hybridization with catalyzed reporter deposition, streptavidin-Nanogold, and silver acetate autometallography: detection of single-copy human paillomavirus. Am J Pathol 1997;150:1553-61.

13. Zehbe I, Rylander E, Strand A, Wilander E. Use of Probemix and Omniprobe biotinylated cDNA probes for detecting HPV infection in biopsy specimens from the genital tract. J Clin Pathol 1993;46:437-40.

14. Berumen J, Casas L, Segura E, Amezcua JL, Garcia-Carranca A. Genome amplification of human papillomavirus types 16 and 18 in cervical carcinomas is related to the retention of E1/E2 genes. Int J Cancer 1994;56:640-5.

15. Reed JA, Nador RG, Spaulding D, Tani Y, Cesarman E, Knowles DM. Demonstration of Kaposi's sarcomaassociated herpes virus cyclin D homolog in cutaneous Kaposi's sarcoma by colorimetric in situ hybridization using a catalyzed signal amplification system. Blood 1998;10:382532.

16. Kerstens HM, Poddighe PJ, Hanselaar AG. A novel in situ hybridization signal amplification method based on the deposition of biotinylated tyramine. J Histochem Cytochem 1995;43:347-52.

17. Sano T, Hikino T, Niwa Y, Kashiwabara K, Oyama T, Fukuda $\mathrm{T}$, et al. In situ hybridization with biotinylated tyramide amplification: detection of human papillomavirus DNA in cervical neoplastic lesions. Mod Pathol 1998;11:19-23. 
18. Saiki RK, Gelfand DH, Stoffel S, Scharf SJ, Higuchi R, Horn GT, et al. Primer-directed enzymatic amplification of DNA with a thermostable DNA polymerase. Science 1988;239:487-91.

19. de Roda Husman AM, Walboomers JM, van den Brule AJ, Meijer CJ, Snijders PJ. The use of general primers GP5 and GP6 elongated at their 3'ends with adjacent highly conserved sequences improves human papillomavirus detection by PCR. J Gen Virol 1995;76:1057-62.

20. Schindl M, Bachtiary B, Dreier B, Birner P, Latinovic L, Breitenecker $\mathrm{G}$, et al. Impact of human papillomavirus infection on the expression of the KAIl metastasis suppressor protein in invasive cervical cancer. Cancer Lett 2001;162:261-6.

21. Karlsen F, Kalantari M, Jenkins A, Pettersen E, Kristensen G, Holm R, et al. Use of multiple PCR primer sets for optimal detection of human papillomavirus. J Clin Microbiol 1996; 34:2095-100.

22. Iwasaki T, Sata T, Sugase M, Sato Y, Kurata T, Suzuki K, et al. Detection of capsid antigen of human papillomavirus (HPV) in benign lesions of female genital tract using anti-HPV monoclonal antibody. J Pathol 1992;168:293-300.

23. Cooper K, Herrington CS, Stickland J, Evans MF, McGee JO. Episomal and integrated human papillomavirus in cervical neoplasia shown by non-isotopic in situ hybridization. J Clin Pathol 1991;44:990-6.
24. Unger ER, Vernon S, Lee DR, Miller DL, Reeves WC. Detection of human papillomavirus in archival tissues: comparison of in situ hybridization and polymerase chain reaction. J Histochem Cytochem 1998;46:535-40.

25. Amortegui AJ, Meyer MP, McIntyre-Seltman K, Locker J. Detection of human papillomavirus DNA in cervical lesions by in situ hybridization using biotinylated DNA probes. Int J Gynecol Pathol 1990;9:306-15.

26. Lie AK, Skjeldestad FE, Hagen B, Johannessen E, Skarsvag S, Haugen OA. Comparison of light microscopy, in situ hybridization and polymerase chain reaction of human papillomavirus in histological tissue of cervical intraepithelial neoplasia. APMIS 1997;105:115-20.

27. Cho NH, Joo HJ, Ahn HJ, Jung WH, Lee KG. Detection of human papillomavirus in warty carcinoma of the uterine cervix: comparison of immunohistochemistry, in situ hybridization and in situ polymerase chain reaction. Pathol Res Pract 1998;194:713-20.

28. Nitta H, Abruzzese R, Coleman M, Keramidas C, Rossing T, Alila H. Visualization of transfected plasmid DNA by in situ hybridization using biotin-labeled plasmid DNA backbone fragment probes and tyramide signal amplification. Cell Vision 1997;5:331-7.

\section{Book Review}

Mohr U, Carlton WW, Dungworth DL, Benjamin SA, Capen CC, Hahn FF: Pathobiology of the Aging Dog, Ames, IA, International Life Sciences Institute, lowa State University Press, 2001 (\$599.95).

Few individuals would belittle the importance of the dog, not only as a companion animal species, but also for clinical, toxicological, and surgical research. Setting aside the vast literature on aging rodents, the dog is perhaps the bestcharacterized species in the science of veterinary pathology. Many veterinary textbooks address diseases and lesions of dogs along with the other domestic animals, but few are focused on the natural diseases of the dog and much less so on the experimental diseases of this species. The textbook Pathobiology of the Aging Dog now fills this void. This two-volume work is designed in a systems approach to cover general diseases and pathology of the dog, but the substance of this text is its basis in research. Indeed, the authors provide not only summary literature reviews on a large number of diseases, but they often provide clinical or biomedical research data on many of these conditions and relate these data comparatively to other species, including man. Most chapters cover aspects of normal and abnormal morphology, pathophysiology, and the biochemistry of many conditions. Supporting figures, photography, and electron micrographs in most chapters are high quality and helpful. Professionals involved in clinical veterinary medicine, clinical veterinary pathology, or medical research involving the canine species would find these volumes useful for evaluating natural canine disease or for providing a frame of reference for lesions that may be induced in the course of research.

\author{
David M. Pinson \\ University of Kansas Medical Center \\ Kansas City, Kansas
}

